\title{
System co-optimization in wireless receiver design with TrACS
}

\author{
Chithrupa Ramesh · Ana Rusu • Mohammed Ismail · \\ Mikael Skoglund
}

Received: 5 December 2007 / Revised: 5 December 2007/ Accepted: 20 May 2008/Published online: 10 July 2008

(C) Springer Science+Business Media, LLC 2008

\begin{abstract}
System co-optimization of the analog receiver front end circuit and the digital baseband processing could enable receiver designs with lower power budgets, as the signal processing in the digital receiver is asymmetric across circuit topologies. This paper presents a simulation tool that could assist with such co-optimized designs. TrACS (Transceiver Architecture and Channel Simulator) is an RF/DSP co-simulator, capable of providing an application-specific system-level perspective to receiver design. The simulator is especially relevant in the context of energy-constrained wireless sensor node design, where the simulator's system perspective determines the compatibility of circuit topologies, modulation techniques and synchronization methods for various wireless scenarios. A few case studies are presented, which illustrate co-optimization of a ZigBEE receiver using TrACS.
\end{abstract}

Keywords Wireless sensor node transceiver . System-level simulator $\cdot$ Co-optimized design tools

C. Ramesh $(\varangle) \cdot$ A. Rusu · M. Ismail · M. Skoglund

Royal Institute of Technology, Stockholm, Sweden

e-mail: cramesh@kth.se
A. Rusu
e-mail: ana@imit.kth.se
M. Ismail
e-mail: ismail@imit.kth.se
M. Skoglund
e-mail: skoglund@ee.kth.se

\section{Introduction}

Wireless sensor networks have found applications in diverse areas, which require robust, efficient and unsupervised operation for long periods of time. Energy in the sensor node is a limited resource [1-3]. The radio transceiver often strains this tenuous resource and therefore ultra low power transceiver designs are proposed to help the node conserve power $[5,6,24]$.

The radio receiver consists of an analog front-end and the digital baseband. The circuit down-converts and digitizes the RF signal and in doing so, impairs the signal with phase noise, cross coupling and intermodulation products [7]. Furthermore, circuits are inherently non-linear but constrained to operate as linear devices. To retain signal integrity, high linearity components with better performance factors are used, at the expense of more power.

The use of shared resources and noisy, fading channels introduces interference, pulse spreading in time and frequency domains, random scaling and additive noises into the desired signal. Complex signal processing techniques such as equalization, error coding, pulse shaping and spread spectrum are required to compensate this degradation [8]. The digital baseband accomplishes all of this.

A power-efficient receiver requires components that consume less power in addition to algorithms with lower computational complexity. The circuit and the baseband processing, together, must meet the system power budget. If the circuit and signal processing are chosen to compliment each other, efficient system level cooperation can be effected. For instance, the use of high resolution Analog to Digital Converters (ADC) can offset the complexity of the error coding technique or vice versa. Similarly, the use of spread spectrum techniques can reduce interference from intermodulation products introduced by nonlinear components. 
These are examples of co-optimization, which is essential for further power conservation. The energy constraint has motivated many cross layer optimization algorithms in the protocol stack $[1,4,9,10,15]$. Co-optimized receiver design takes this concept further by innovating cohesive circuit and signal processing in an optimum receiver. WINS [11], WiseNET [23] and PicoRadio [12] are examples of cooptimized sensor node design.

However, tools that permit such designs are not readily available. There are circuit simulators, such as Cadence ${ }^{\circledR}$, pSpice ${ }^{\circledR}$, ADS $^{\circledR}$ and digital baseband processing simulators such as the BER tool in MatLab ${ }^{\circledR}$. Very few simulators combine both aspects of the receiver-the analog front end and the digital baseband. ADS Ptolemy [13] provides a simulation of the total communications system path, which helps evaluate system performance of an integrated circuit. Even this tool cannot be used for co-optimized design, as the system performance must be projected before commencing circuit design. TrACS is an RF/DSP co-simulator, designed to fill this gap [14]. Due to its abstract circuit models, TrACS is capable of offering a design preview along with system performance measures, thus permitting the selection of co-optimal circuit architectures and baseband processing techniques for any given wireless scenario.

The rest of this article is organized as follows. Section 2 presents the motivation and guiding principal behind TrACS. Section 3 presents components of TrACS and their implementation. A few case studies are presented in Sect. 4 to illustrate the capabilities of TrACS in co-optimization of a ZigBEE receiver design. This is followed by the conclusions in Sect. 5.

\section{Concept of TrACS}

Co-optimization involves an exploration of complimentary circuit and signal processing techniques from a system perspective. The combined parameter space of circuit design and signal processing is large and optimal solutions cannot be found analytically. This task is best performed by a simulator capable of modeling circuits and executing baseband processing algorithms. This is the motivation for the concept of TrACS-a Transceiver Architecture and Channel Simulator. The simulation of the channel along with the receiver permits the selection of an applicationspecific solution.

TrACS contains abstracted models of the receiver front end circuit. These circuits implement the down-conversion through various topologies and a few, such as the Low-IF and Direct Conversion topologies are well identified for low-power circuits. These are included in TrACS, along with the class of sub-sampling receivers, which is an active area of research. Each of these topologies affects the signal in different ways and these have been identified and modeled as described in Sect. 3.

The wireless channel and the digital baseband are modeled in Matlab. Channel models include the theoretical AWGN (Additive White Gaussian Noise) channel and fading channels. Baseband processing techniques such as pulse shaping, spread spectrum and modulation are included. The recovery of unknown channel parameters such as phase, frequency and timing are simulated as well, and synchronization errors can be modeled using TrACS.

TrACS uses the baseband equivalent model (BEM), where the complex baseband equivalent is used to represent the RF signal. This amounts to removing the frequency content of the signal and modeling the complex envelope to run quicker simulations. However, the RF signal in the receiver front end is bandpass and is translated to the baseband in TrACS.

The simulator provides a cohesive platform for systemlevel verification of RF circuit design, as presented in [14]. Along with the circuit design, digital receiver algorithms for power conservation and signal compensation can be tested simultaneously to determine the effect on the BER (Bit Error Rate) of the system. TrACS aids the circuit designer in choosing the receiver architecture given the PHY layer specifications and the wireless scenario. The simulator can also help reverse this study-given the circuit parameters for minimum performance of a receiver and the wireless scenario, a set of optimal radio specifications can be found. Finally, TrACS could help identify optimal solutions in a test bed for wireless sensor networks.

\section{Components of $\operatorname{TrACS}$}

TrACS models the analog front end receiver, digital baseband, synchronization and application specific wireless scenarios in Matlab. The usage of TrACS as a tool is also outlined below.

\subsection{Receiver architectures}

Low power radio design is an active area of research, with a few receiver architectures well identified for their performance, i.e., Low-IF, Direct Conversion [5, 6] and IF Sub-Sampling [27]. The baseband equivalent model (BEM), shown in Fig. 1, is used to represent the signal. Each component in the receiver contributes to the signal impairment, and this error source is modeled in the baseband. Before we discuss the circuit topologies in detail, it is important to mention that non-linear systems cause harmonics, gain compression and intermodulation quantified by the performance metrics $\mathrm{IP}_{2}$ and $\mathrm{IP}_{3}$ [7]. 
Fig. 1 Complex baseband equivalent model

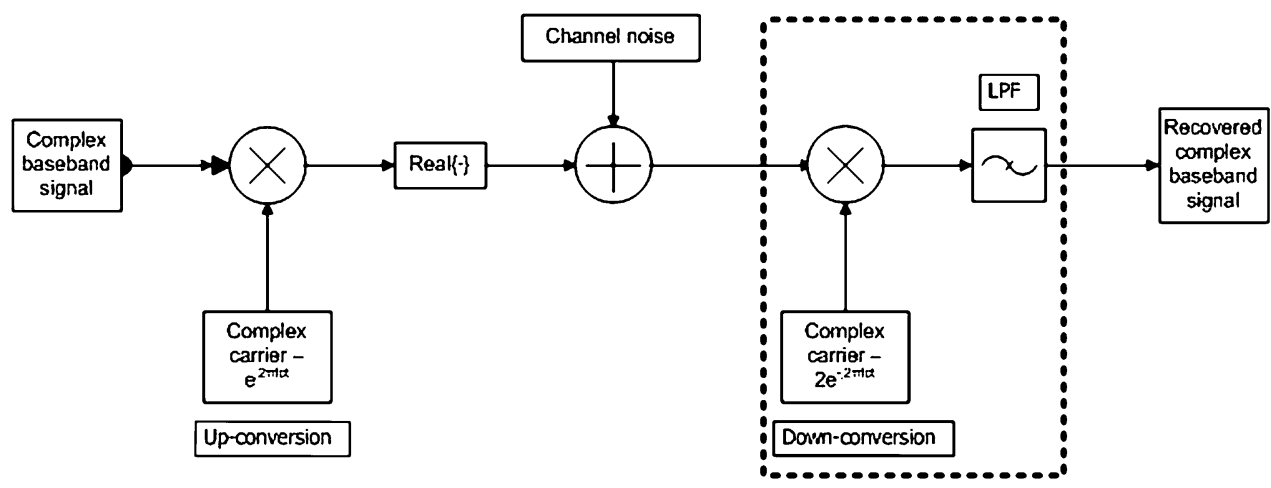

\subsubsection{Low-IF topology}

This is the superheterodyne architecture with a lower intermediate frequency (IF between $1 \mathrm{MHz}$ and $10 \mathrm{MHz}$ ). The Low-IF topology, shown in Fig. 2, simplifies the IF filter design but the image filter requirements become steep. The Low-IF uses a quadrature receiver to perform image cancellation.

The components of this topology and error sources are discussed below. The signal is amplified by the LNA (Low Noise Amplifier) gain, $\alpha$. Non-linearities in the circuit introduce intermodulation products, which may saturate the circuit. As the second order products are filtered out by the IF filter, only the third order products are modeled here. This signal is at the intermodulation frequency $\left(f_{\mathrm{IM}}\right)$ and is modeled in baseband after down-conversion and low pass filtering (Eq. 1).

$$
\begin{aligned}
A_{\mathrm{IM} 3} \cos \left(\omega_{\mathrm{IM}} t\right) \times 2 e^{-j \omega_{\mathrm{LO}} t}= & A_{\mathrm{IM} 3}\left[\frac{e^{j \omega_{\mathrm{IM}} t}+e^{-j \omega_{\mathrm{IM}} t}}{2}\right] \\
& \times 2 e^{-j \omega_{\mathrm{LO}} t} \\
= & A_{\mathrm{IM} 3} e^{j\left(\omega_{\mathrm{IM}}-\omega_{\mathrm{LO}}\right) t}
\end{aligned}
$$

$A_{\mathrm{IM} 3}=\alpha \frac{A_{\mathrm{in}}^{3}}{A_{\mathrm{IP}_{3}}^{2}}$

where $A_{\text {in }}$ is the input amplitude of the signal, $\alpha$ is the LNA Gain and $A_{\mathrm{IP}_{3}}$ is the input $\mathrm{IP}_{3}$.

The local oscillator may not be perfectly tuned to the carrier frequency, and this causes errors. Reciprocal Mixing refers to the error that occurs due to the non-ideal impulse characteristic of the LO output. This can be modeled as a flat noise of power $\left(\mathrm{S}_{0} \cdot \Delta \mathrm{B}\right)$, where $\mathrm{S}_{0}$ is the constant power spectral density of the unwanted signal over the bandwidth of the signal of interest $(\Delta \mathrm{B})$. Phase noise of a local oscillator also distorts the phase of the carrier. This leads to constellation rotation and spreading and is modeled by multiplying the signal with $\mathrm{e}^{\mathrm{j} \varphi}$, where $\varphi$ is a random phase between $[-\pi, \pi]$. The frequency transformation does not affect either of these errors.

The mixer down-converts the signal to the IF band. As the mixer does not preserve the polarity of the difference between its two input frequencies, it causes an image frequency $\left(\omega_{\mathrm{LO}}-\omega_{\mathrm{IF}}\right.$ or $\left.\omega_{\mathrm{LO}}+\omega_{\mathrm{IF}}\right)$ to also down-convert to the same IF band. The interference is reduced by image cancellation but never completely because of imbalances in

Fig. 2 Low-IF architecture

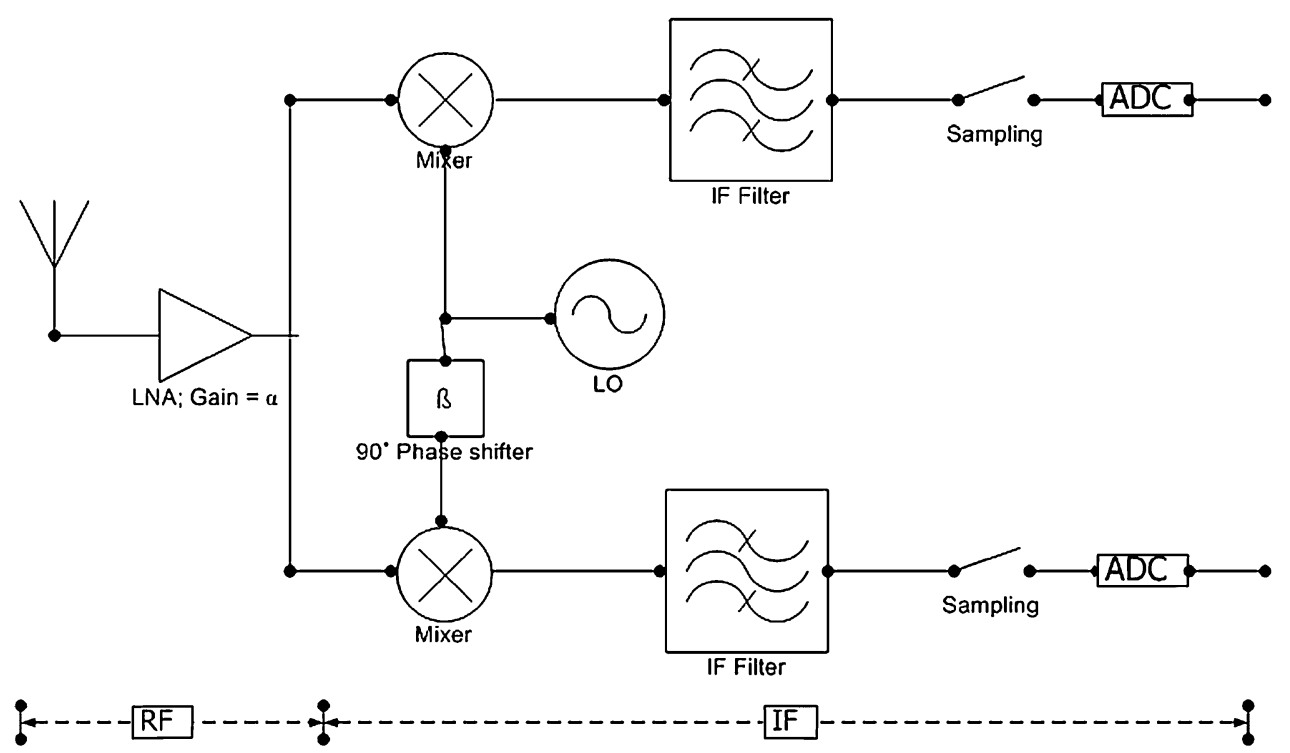


the quadrature arms. A diminished power image signal is added to the RF signal to model this phenomenon. Sampling jitter corrupts the signal and this distortion is modeled with flat noise of variance $P_{\mathrm{j}}$, given by $P_{j}=2 \pi^{2} f_{\mathrm{in}}^{2} A^{2} \sigma_{j}^{2}$ where $f_{\text {in }}$ is the signal frequency, $A$ is the signal amplitude and $\sigma_{\mathrm{j}}$ is the standard deviation of the jitter process [25, 26]. Finally, the ADC introduces a quantization error, which lies uniformly between $[-\Delta / 2, \Delta / 2]$ and $\Delta$ is the step-size of the quantizer. The Low-IF model is shown in Fig. 3.

\subsubsection{Direct conversion architecture}

Figure 4 shows the zero-IF architecture where the signal is directly down-converted to the baseband. Simplicity of design and the lack of need for an image-reject filter are some of the advantages of this topology. A quadrature receiver is used to cancel the image.

The components of this topology and error sources are discussed below. The signal is amplified by the LNA gain, $\alpha$. Intermodulation products cause interference. As the third order products are filtered out by the low pass filter, only the second order products are modeled here. This signal appears at the intermodulation frequency $\left(f_{\mathrm{IM}}\right)$ and the frequency transformation and low pass filtering must be applied to it. The implementation is the same as in (1) with $A_{\text {IM3 }}$ replaced by

$A_{\mathrm{IM} 2}=\alpha \frac{A_{\mathrm{in}}^{2}}{A_{\mathrm{IP} 2}}$

The local oscillator errors are modeled as before. The signal is its own image and a quadrature scheme is used to isolate the side bands, but imbalances in the arms of the circuit can lead to incomplete isolation. This phenomenon, known as IQ Imbalance, is modeled with parameters $\varepsilon$, representing the amplitude imbalance, and $\varphi$, the phase imbalance, as shown below.

$$
\begin{aligned}
\operatorname{Real}\{r\}= & \operatorname{Real}\{r\} \cdot\left(1+\frac{\varepsilon}{2}\right) \cdot \cos \left(\frac{\theta}{2}\right)-\operatorname{Imag}\{r\} \\
& \cdot\left(1+\frac{\varepsilon}{2}\right) \cdot \sin \left(\frac{\theta}{2}\right)
\end{aligned}
$$

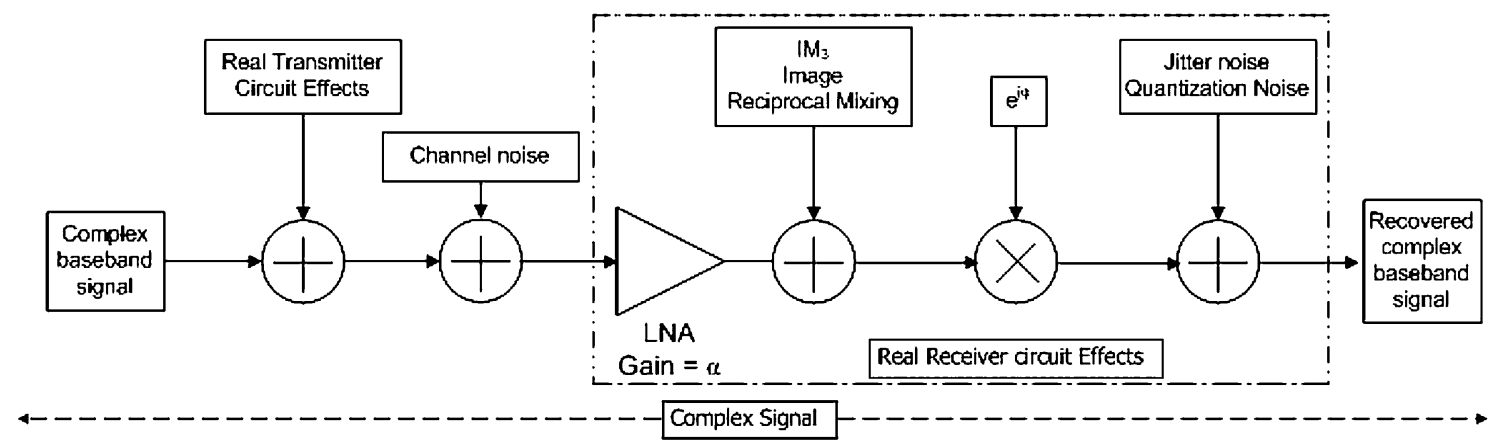

Fig. 3 Model of the Low-IF architecture

Fig. 4 Direct conversion architecture

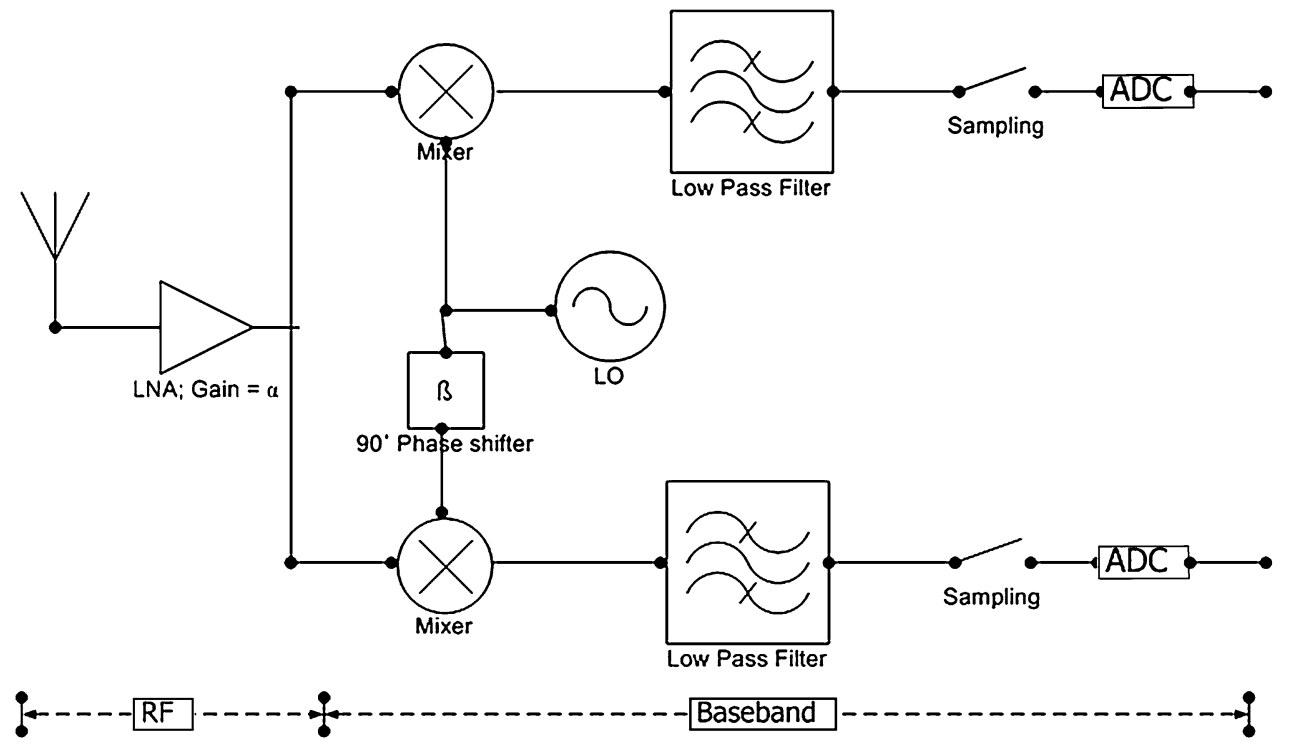




$$
\begin{aligned}
\operatorname{Imag}\{r\}= & -\operatorname{Real}\{r\} \cdot\left(1-\frac{\varepsilon}{2}\right) \cdot \sin \left(\frac{\theta}{2}\right)+\operatorname{Imag}\{r\} \\
& \cdot\left(1-\frac{\varepsilon}{2}\right) \cdot \cos \left(\frac{\theta}{2}\right)
\end{aligned}
$$

The signal is in the baseband and stray DC offsets in the circuit add to the desired signal. DC offset from three main sources is modeled. (a) Self-mixing of local oscillator signals leaking into the RF port of the mixer and the input port of the LNA. This is modeled as $\mathrm{K}_{1}+\mathrm{A} \cdot \mathrm{K}_{2}$; where $\mathrm{K}_{1}$ is the attenuation of the LO signal leaking into the mixer, $\mathrm{K}_{2}$ is the attenuation of the LO signal leaking into the LNA and A is the LNA gain factor. (b) Self-mixing of LO signals leaking into, radiated from and reflected back to the antenna. This is modeled with a complex sinusoid at the Doppler shifted frequency $\left(f_{\mathrm{DS}}\right)$ of amplitude $\mathrm{A} \cdot \mathrm{K}_{3}$; where $K_{3}$ is the attenuation of the LO signal leaking into the antenna. (c) Self-mixing of strong in-band interferers leaking into the LO port of the mixer from the output of the LNA. This is modeled with a complex sinusoid at the difference frequency between in-band interferer and carrier frequency $\left(f_{\mathrm{O}}\right)$ of amplitude $\mathrm{A} \cdot \mathrm{K}_{4}$; where $\mathrm{K}_{4}$ is the attenuation of interfering signal leaking into the LO port of the mixer from the output of the LNA [22].

The circuit exhibits a low-frequency noise known as flicker noise or $1 / f$ noise. This is represented as random white noise of a user-input power level in the baseband. The sampling jitter and quantization error are modeled as before. The Direct Conversion model is shown in Fig. 5.

\subsubsection{IF sub-sampling architecture}

The intermediate frequency in the Low-IF architecture is still high and makes ADC implementation difficult. One possible approach is to down-sample the bandpass signal but the reduction in sampling frequency comes at the cost of aliasing noise. This topology is shown in Fig. 6.
The components of this model and the error sources are the same as described above for the Low-IF architecture. The only addition is the aliasing noise due to inadequate anti-alias filtering. To model the aliasing noise, we look at down sampling in detail in Fig. 7. The bandpass signal bandwidth is $\mathrm{B}$, the anti-aliasing filter bandwidth is $\mathrm{B}_{\mathrm{f}}$ and the sampling frequency is $f_{s}$. The noise within the filter bandwidth is folded over many times at each folding frequency $\left(f_{\text {fold }}\right)$ to cause the aliasing noise, as in (6). The total noise is the noise floor of the analog signal multiplied by the number of times the filtered signal is folded over. Let this number be $n_{\text {fold }}$, given in (7). The baseband equivalent model for this topology is summarised in Fig. 8.

$$
\begin{aligned}
& f_{\text {fold }}=f_{c}+\frac{B}{2}-\frac{f_{s}}{4}+\frac{B}{2} ; \\
& f_{\text {fold }}=\left\{\begin{array}{l}
\left(f_{c}-\frac{f_{s}}{4}+B\right) f_{s}>2 B \\
\left(f_{s}+\frac{B}{2}\right) \quad f_{s}=2 B
\end{array}\right. \\
& n_{\text {fold }}=\frac{f_{c}+\frac{B_{f}}{2}-f_{\text {fold }}}{\frac{f_{s}}{2}}+1 ; \\
& n_{f o l d}=\left\{\begin{array}{l}
\left(\frac{B_{f}}{f_{s}}-\frac{2 B}{f_{s}}+\frac{3}{2}\right) f_{s}>2 B \\
\left(\frac{B_{f}}{f_{s}}+\frac{1}{2}\right) \quad f_{s}=2 B
\end{array}\right.
\end{aligned}
$$

\subsection{Digital baseband}

The digital baseband signal processing modeled here includes modulation, pulse shaping and spreading. Pulse shapes such as rectangular, root raised cosine and half sine pulse are included in TrACS. Spread Spectrum is included in the baseband model using the Direct Sequence Technique. With the inclusion of synchronization effects, Frequency Hopped systems can be simulated as well.

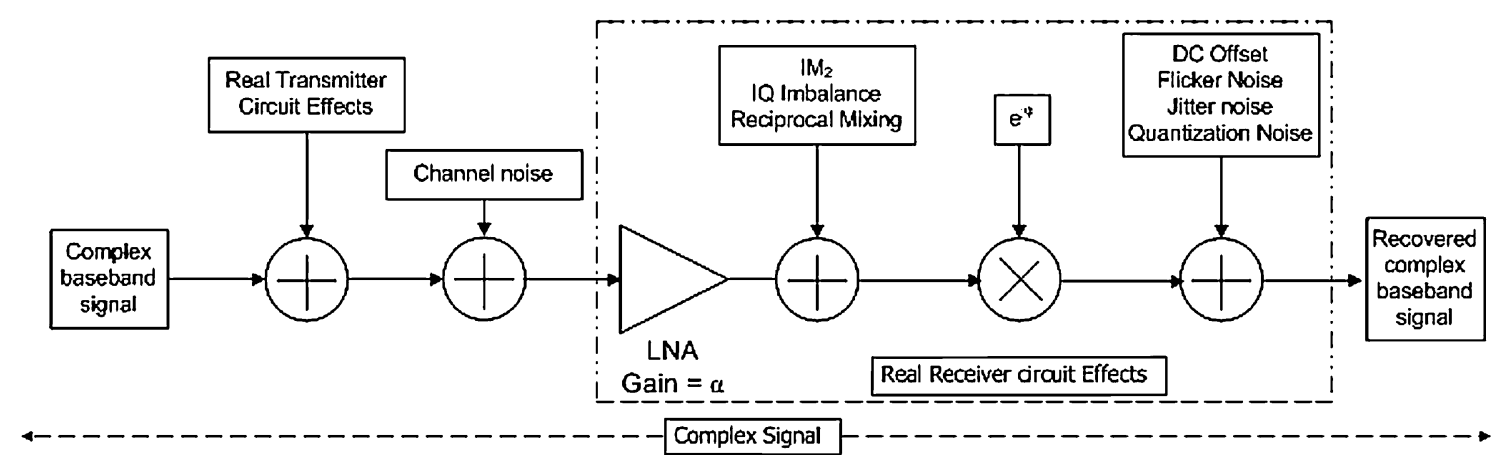

Fig. 5 Model of the direct conversion architecture 
Fig. 6 IF sub-sampling architecture
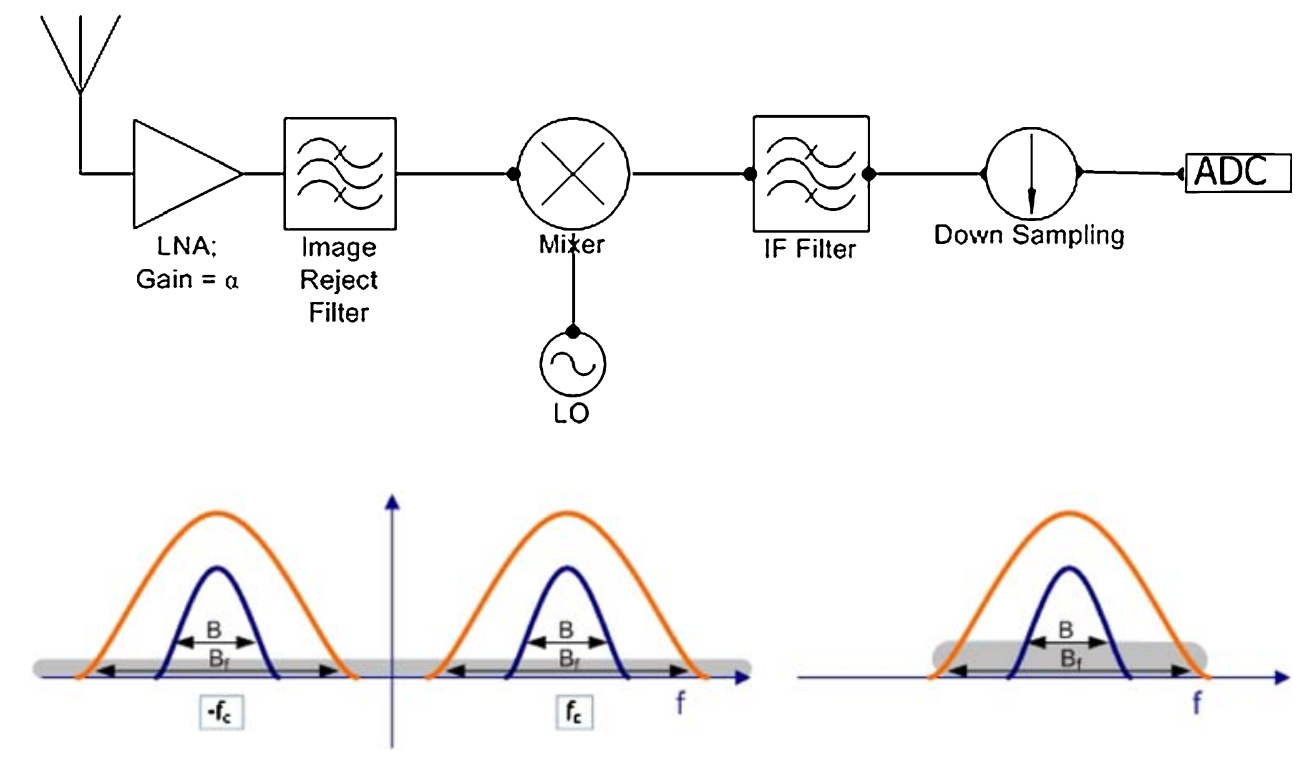

Fig. 7 Aliasing noise due to insufficient anti-alias filtering

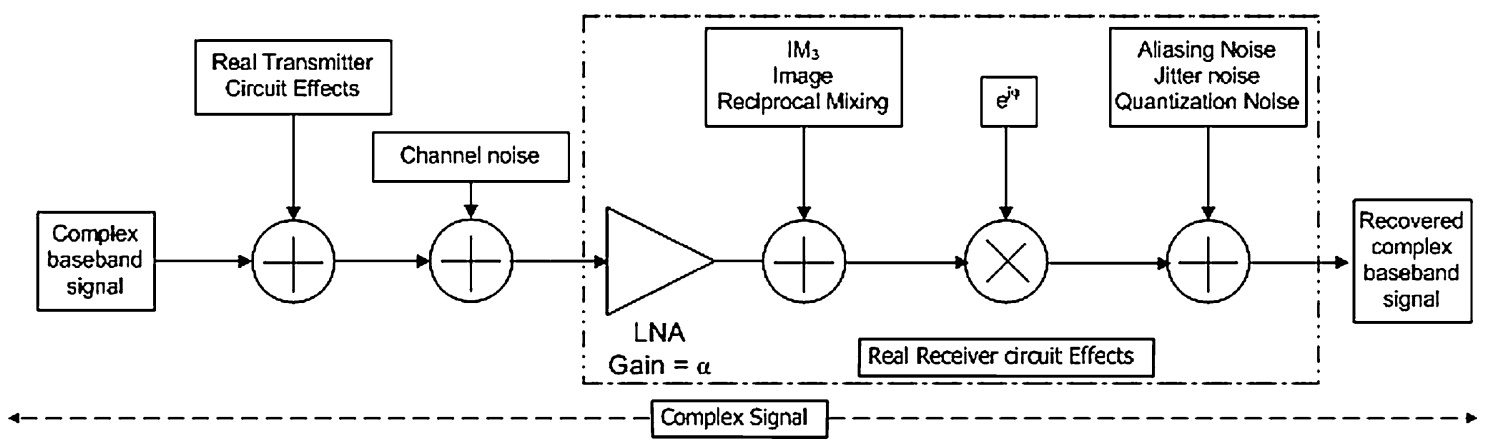

Fig. 8 Model of the IF sub-sampling architecture

\subsubsection{Modulation techniques}

There exists an inherent trade off between power-hungry modulation schemes and power hungry detection circuits. The most efficient modulation schemes (in terms of power) are BPSK (Binary Phase Shift Keying) or QPSK (Quadrature Phase Shift Keying). But, the demodulation circuits require precise synchronization for time, frequency and phase and all of these operations increase the power budget of the receiver. On the other hand, non-coherent Frequency Shift Keying (FSK) and On-Off Keying (OOK) can be detected without the use of an $\mathrm{ADC}$, and the receiver power requirements are very low.

While analysis of circuit requirements and detection theory do provide us power estimates, only a simulator can deliver actual performance estimation. Thus, we have implemented OOK, FSK and phase shift schemes in TrACS with the purpose of studying the trade off in power requirements. The phase shift schemes implemented are BPSK, QPSK, O-QPSK (offset QPSK), DPSK (differential PSK) and D-QPSK. Options for coherent and non-coherent demodulation are available for FSK, while differential coding is also available for the phase modulation schemes.

\subsection{Synchronization}

Exploring co-optimal receiver topologies and digital baseband techniques would not yield meaningful results if the problem of synchronization were ignored. The receiver assumes perfect channel knowledge and synchronization. However, synchronization and channel estimation are practical problems that must be solved in every receiver and these require dedicated circuits and algorithms, which consume power $[15,16,18-21]$. Optimal receiver solutions must include this power drain in the system budget. TrACS models synchronization, estimation and tracking errors to generate more realistic simulations. Typically, receivers first compensate frequency offset using non-data aided and non-clock aided algorithms. This is followed by clock recovery, symbol timing estimation and phase correction, all of which generate errors that are simulated in TrACS. 
Table 1 List of wireless scenarios

\begin{tabular}{|c|c|}
\hline Channel & Scenario \\
\hline AWGN & $\begin{array}{l}\text { A theoretical model and a non-fading channel, } \\
\text { used for verification. }\end{array}$ \\
\hline Slow, flat fading & Stationary, outdoor scenario. \\
\hline Slow, FS fading & $\begin{array}{l}\text { Stationary, indoor scenario or a slow-traffic } \\
\text { urban area. }\end{array}$ \\
\hline Fast, flat fading & High-speed outdoor scenario. Eg. Expressway. \\
\hline Fast, FS fading & Urban/downtown areas with high speed traffic. \\
\hline
\end{tabular}

FS: frequency selective

\subsection{Wireless scenarios}

TrACS defines various application scenarios based on the wireless channel used. Table 1 lists the channels and corresponding real life scenarios.

\subsection{Usage of TrACS}

The user is presented with a graphical interface to interact with TrACS. The interface accepts inputs for signal, receiver circuit, wireless channel and synchronization parameters. The user can also choose the baseband specifications. The results of the simulator are circuit level Chip Error Rate curves, obtained after the digitization of the signal and the system level Symbol Error Rate curve, obtained after detection of the signal [14].

\section{Case studies}

The following case studies illustrate benefits of TrACS from different perspectives. These simulations use the input data presented in Tables 2-5.

\subsection{Choosing a circuit topology}

TrACS can be used to choose the optimum circuit architecture, given the baseband specifications. Figure 9 is the Bit Error Rate curve (BER versus $\mathrm{E}_{\mathrm{b}} / \mathrm{N}_{\mathrm{o}}$ ) for non-coherent FSK in a slow, frequency selective fading channel. The performance of three circuit topologies at the system level are compared and TrACS estimates Direct Conversion to be the optimum architecture for this scenario.

\subsection{Comparing modulation techniques}

TrACS can be used to find the optimum baseband specifications, such as the modulation technique to be used, given the application scenario. This is useful in identifying the baseband to use given multiple options, as in the ISM band. It could also be a useful design feature for cognitive
Table 2 Circuit parameters

\begin{tabular}{ll}
\hline Parameter & Value \\
\hline Receiver Sensitivity & $-110 \mathrm{dBm}$ \\
LNA Gain & $20 \mathrm{~dB}$ \\
$\mathrm{IIP}_{2}, \mathrm{IIP}_{3}$ & $25,-15 \mathrm{dBm}$ \\
Intermodulation product frequency & $500 \mathrm{kHz}$ \\
LO phase noise- $\mathrm{S}_{0}$ & $-109 \mathrm{dBc} / \mathrm{Hz}$ \\
LO phase noise bandwidth- $\Delta \mathrm{B}$ & $1 \mathrm{MHz}$ \\
LO phase angle distortion & $0.1 \mathrm{Radians}$ \\
Image retention factor & 0.1 \\
IQ amplitude imbalance- $-\varepsilon$ & 0.1 \\
IQ phase imbalance- $\theta$ & 0.5 \\
Flicker noise power & $-100 \mathrm{dBm}$ \\
DC offset leakage coefficients $\mathrm{K}_{1}, \mathrm{~K}_{2}-\mathrm{K}_{4}$ & $-55 \mathrm{~dB},-40 \mathrm{~dB}$ \\
Doppler shifted frequency- $f_{\mathrm{DS}}$ & $100 \mathrm{kHz}$ \\
Down-converted interferer frequency & $2 \mathrm{MHz}$ \\
Aliasing filter bandwidth & $4 \mathrm{MHz}$ \\
RF Sampling Frequency & $4 \mathrm{MHz}$ \\
Maximum signal frequency at $\mathrm{S} / \mathrm{H}$ & \\
$f_{\text {LOIF,in }}, f_{\mathrm{DC} \text {,in }}, f_{\mathrm{IFSS}, \text { in }}$ & $2 \mathrm{MHz}, 500 \mathrm{kHz}, 5 \mathrm{MHz}$ \\
Std deviation of sampling jitter $\left(\sigma_{\mathrm{j}}\right)$ & $10-7$ \\
Quantizer resolution & $12 \mathrm{bits}$ \\
\hline
\end{tabular}

Table 3 Signal parameters

\begin{tabular}{ll}
\hline Parameter & Value \\
\hline Data rate & $40 \mathrm{kbps}$ \\
Bandwidth & $2 \mathrm{MHz}$ \\
Processing gain & 15 or 8 \\
Chip length & $1.667 \mu$ s or $3.125 \mu \mathrm{s}$ \\
Oversampling ratio & 8 or 16 \\
\hline
\end{tabular}

Table 4 Channel parameters

\begin{tabular}{|c|c|}
\hline Parameter & Value \\
\hline Doppler shift $\left(f_{\mathrm{D}}\right)$ & $250 \mathrm{~Hz}$ \\
\hline Rician factor, $\mathrm{K}$ & $10 \mathrm{~dB}$ \\
\hline Path delay-flat fading & {$\left[\begin{array}{lll}0 & \left.1 / 100 \cdot\left(\mathrm{T}_{\mathrm{c}} / \mathrm{Q}\right)\right]\end{array}\right.$} \\
\hline Path delay-FS fading & {$\left[\begin{array}{lll}0 & 10 & \left(\mathrm{~T}_{\mathrm{c}} / \mathrm{Q}\right)\end{array}\right]$} \\
\hline Path gain-flat fading & {$[0-20]$} \\
\hline Path gain-FS fading & {$[0-10]$} \\
\hline
\end{tabular}

Table 5 Synchronization parameters

\begin{tabular}{ll}
\hline Parameter & Value \\
\hline Frequency error & $0.1 \% f_{c}$ \\
Symbol timing error & $0.1 \% \mathrm{~T}_{\mathrm{c}}$ \\
Phase error & $0.1 \pi$ \\
\hline
\end{tabular}


Fig. 9 Optimum circuit architectures for non-coherent FSK in a slow, flat fading channel

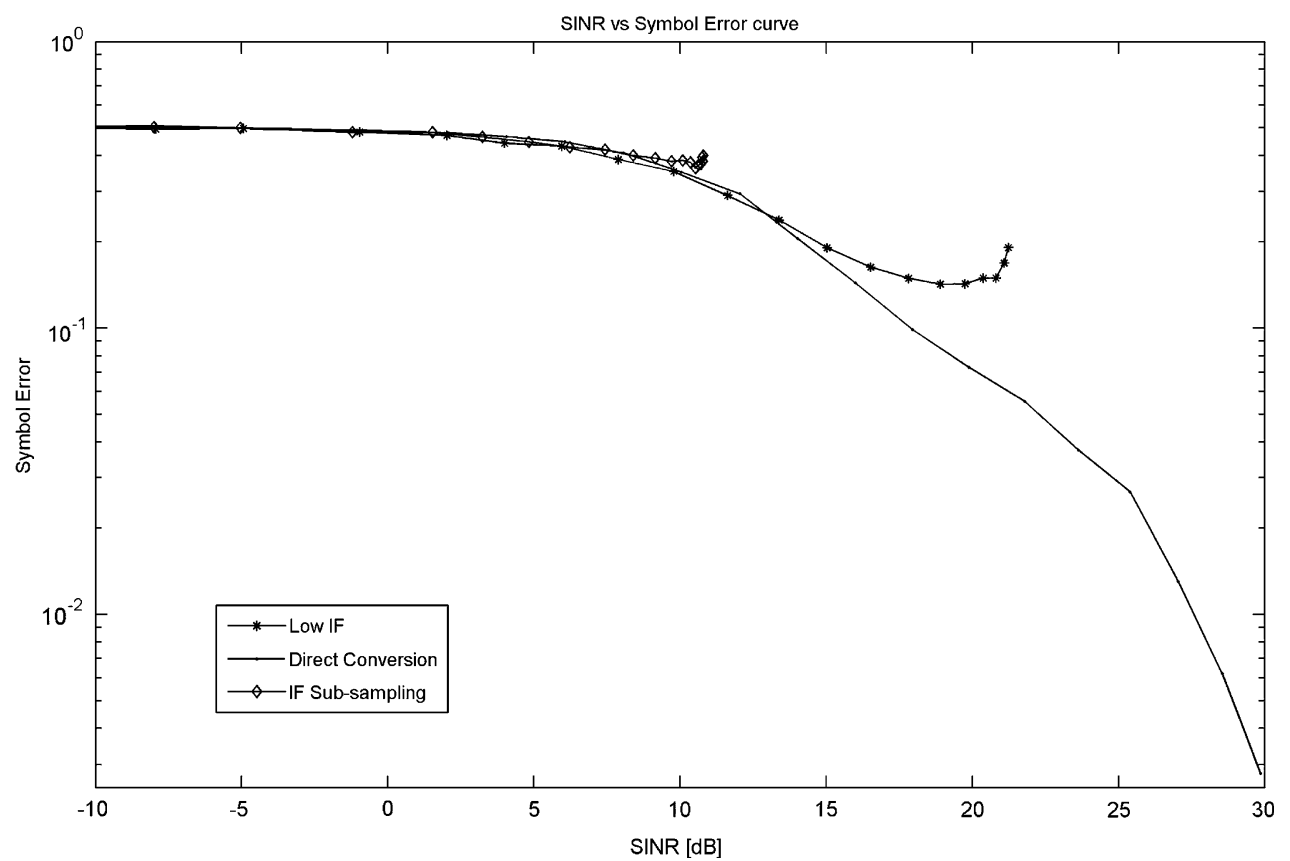

radios. Figure $10(\mathrm{a}, \mathrm{b})$ compares the performance of O-QPSK and differentially encoded BPSK in a Slow, Frequency Selective Rayleigh Fading Channel using Direct Conversion and Low-IF architectures respectively. It can be noted that O-QPSK performs better than differentially encoded BPSK in both receiver topologies.

\subsection{Performance with estimation errors}

The results shown in Fig. 10 are inconclusive as the performance penalty due to synchronization, estimation and tracking errors is not recorded. Figure 11 presents the same comparison with synchronization and estimation errors, using a Direct Conversion architecture alone [16]. It can be observed that O-QPSK is less affected by these errors than differentially encoded BPSK. This is an important factor in dispersive channels, where phase estimation must be used to recover the data.

\subsection{ZigBee receiver design}

ZigBee has been proposed as the communication standard for wireless sensor networks. It is built on the PHY and MAC Layers of the IEEE Standard 802.15.4 [17]. IEEE 802.15.4 provides a dual PHY Layer $(902-928 \mathrm{MHz}$ and the $2.4 \mathrm{GHz}$ ISM band) with different baseband specifications for each of these bands. This provides us with the perfect opportunity to verify the co-optimization capacity of TrACS. The task is to design a low power radio receiver for various application scenarios using the ZigBee standard. In this case study, we use TrACS to identify the co-optimized receiver design - the digital baseband and receiver architecture which perform best together for the four application scenarios listed in Table 1.

The results are presented in Table 6 . These results are based on the inputs used in Tables 2-5 and can vary depending on the selected parameters. The better design option has been highlighted, when a significant performance difference has been noted. The results indicate a preference towards the $900 \mathrm{MHz}$ band or differentially encoded BPSK only in fast fading channels. This is due to the penalty paid by a coherent receiver (O-QPSK) in rapidly varying channels. The designer may still choose to use the $2.4 \mathrm{GHz}$ band for its larger bandwidth, and relatively lower congestion. These results are indicative, but not conclusive. However, TrACS provides the RF designer a quick preview of the system performance of all possible design alternatives, permitting him to make an informed choice without the need for prolonged study.

\section{Conclusions}

TrACS provides a common platform for analog front end receiver modeling and digital baseband processing, with emphasis on techniques used in wireless sensor nodes. Optimizing for performance with a power constraint requires a system perspective to explore compatible receiver topologies, digital baseband techniques and synchronization methods. TrACS can be used to identify optimally compatible receivers for application specific scenarios. The simulator's system-level performance was 
Fig. 10 Comparison of ZigBEE baseband for a (a) direct conversion (b) Low-IF receiver in a slow, frequency selective fading channel

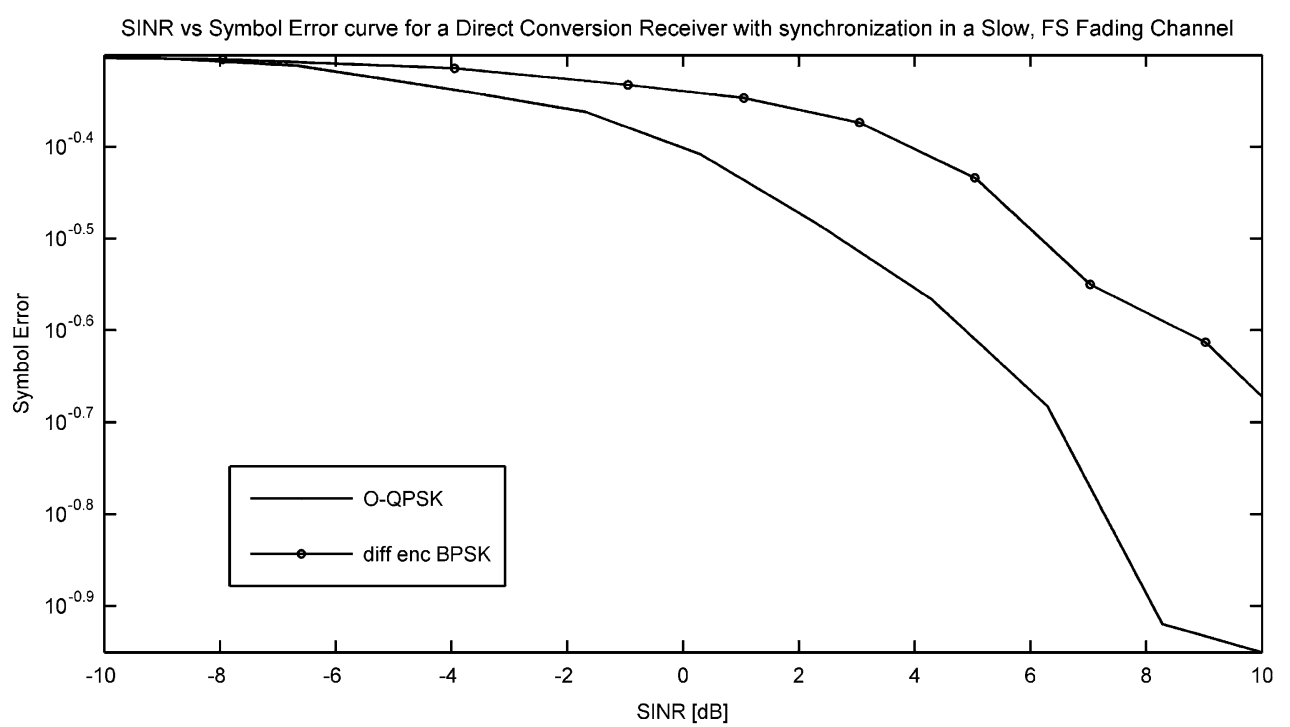

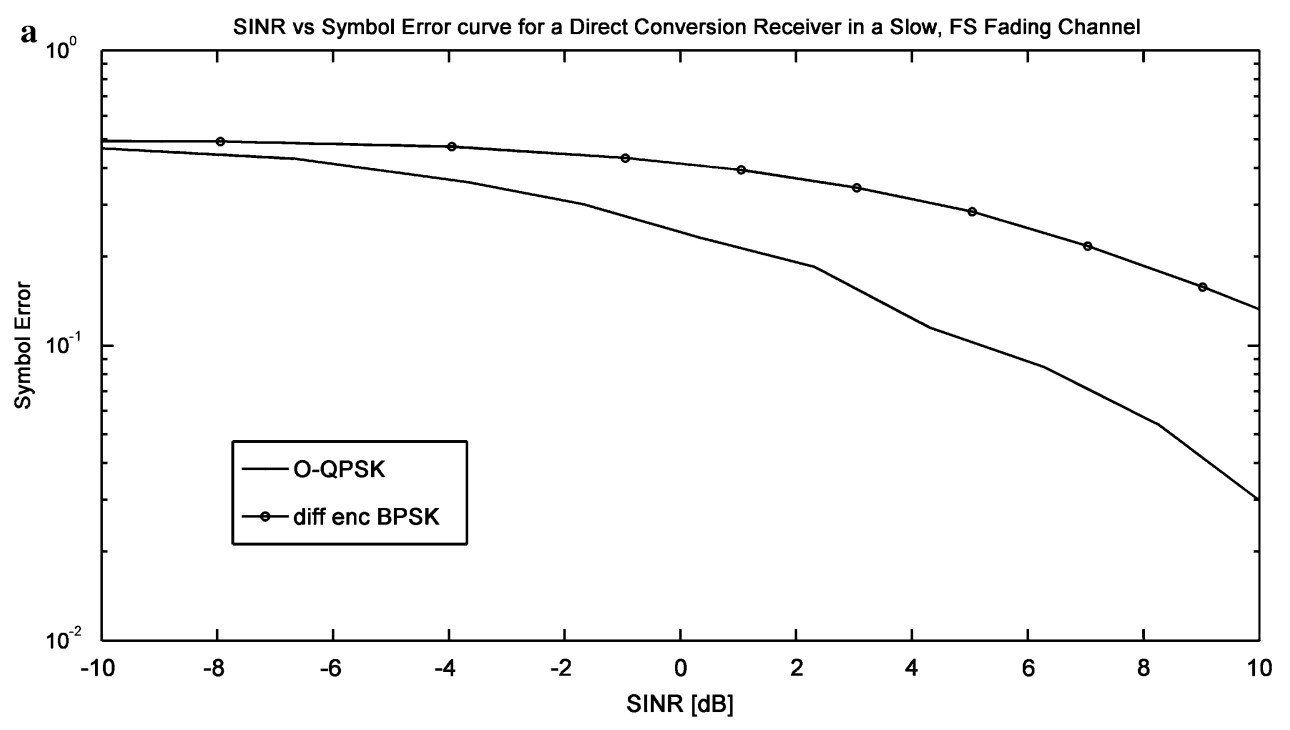

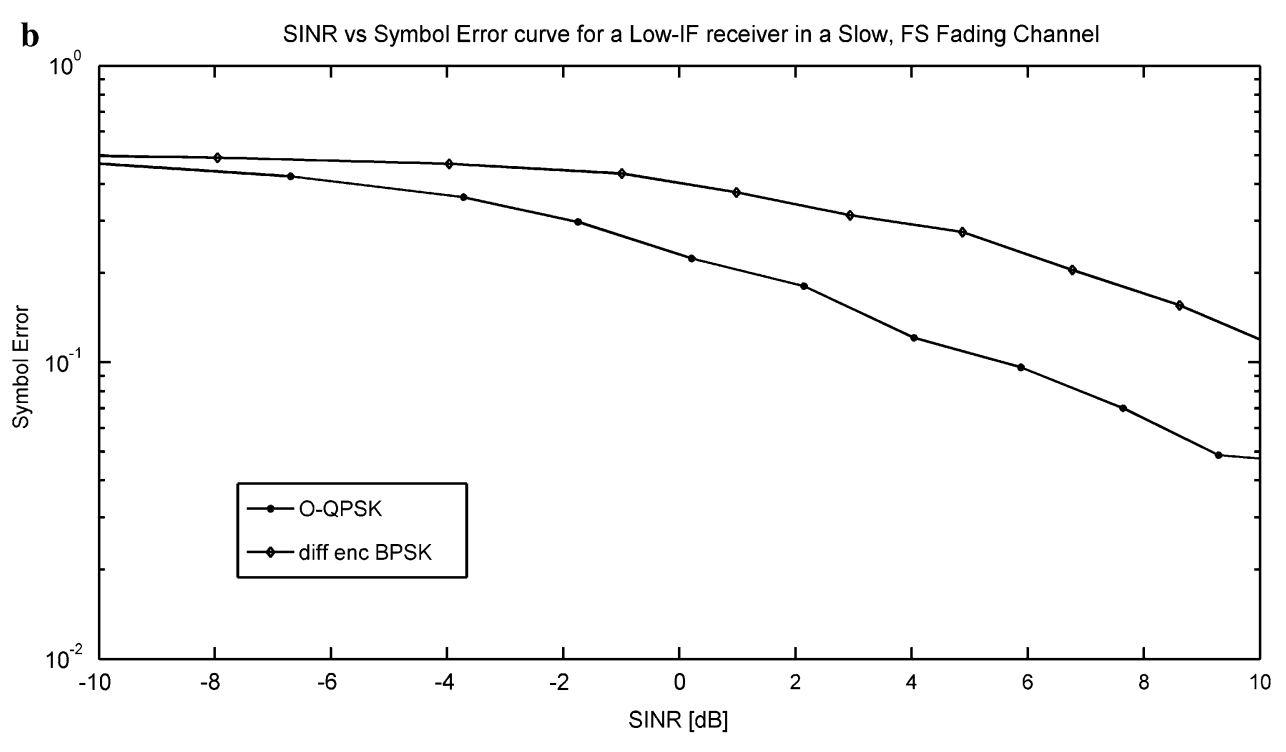

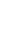

Fig. 11 Comparison of ZigBEE baseband for a direct conversion receiver with synchronization errors in a slow, frequency selective fading channel 
Table 6 Application-specific co-optimized low-power receiver designs

\begin{tabular}{lll}
\hline Application scenario & $902-928 \mathrm{MHz}$ & $2.4 \mathrm{GHz}$ \\
\hline $\begin{array}{l}\text { Stationary, outdoor } \\
\text { scenario. }\end{array}$ & $\begin{array}{c}\text { Direct conversion/ } \\
\text { low IF }\end{array}$ & Direct conversion \\
$\begin{array}{l}\text { Stationary, indoor } \\
\text { scenario or a }\end{array}$ & Direct conversion & $\begin{array}{c}\text { Direct conversion/ } \\
\text { low IF }\end{array}$ \\
slow-traffic & & \\
urban area. & & Direct conversion/ \\
High-speed outdoor & Low IF & low IF \\
scenario. Eg. & & Direct conversion/ \\
expressway. & & low IF \\
$\begin{array}{l}\text { Urban/downtown } \\
\text { areas with high }\end{array}$ & Direct conversion/ & \\
speed traffic. & low IF & \\
\hline
\end{tabular}

verified through case studies, which matched wellestablished results, while offering a new and useful perspective at the system level. The simulator uses this system perspective to identify the ZigBEE baseband and the most compatible receiver architecture for a number of application scenarios. This illustrates the capability of TrACS to co-optimize wireless sensor node receiver design.

System co-optimization of the analog receiver front end circuit and the digital baseband processing is an interdisciplinary approach and tools to aid such designs are not easy to come by. TrACS can fill this niche and contribute to the ultra low power design paradigm for wireless sensor node receivers.

Acknowledgments This work has been funded by Wireless@KTH and the Swedish Foundation for Strategic Research under the RaMSiS project.

\section{References}

1. Goldsmith, A. J., \& Wicker, S. B. (2002). Design challenges for energy-constrained ad hoc wireless networks. IEEE Wireless Communications, 9(4), 8-27.

2. Sadler, B. M. (2005). Fundamentals of energy-constrained sensor network systems. IEEE A\&E Systems Magazine, 20(8), 17-35. Part 2: Tutorials-Sadler.

3. Chong, C. Y., \& Kumar, S. P. (2003). Sensor networks: Evolution, oppurtunities and challenges. Proceedings of the IEEE, 91(8), 1247-1256.

4. Thoonen, G., Lopelli, E., van der Tang, J., \& van Roermund, A. System level considerations for Ultra-low power transmitter-only wireless networks in the indoor environment. w3.ele.tue.nl/fileadmin/ele/ICS/MsM/Files/Lopelli/Prorisc_Thoonen.pdf.

5. Crols, J., \& Stayeart, M. S. J. (1998). Low-IF topologies for high performance analog front ends of fully integrated receivers. IEEE transactions on circuits and systems-II. Analog and Digital Signal Processing, 45(3), 269-282.
6. Lin, T. H., Kaiser, W. J., \& Pottie, G. J. (2004). Integrated lowpower communication system design for wireless sensor networks. Communications Magazine, IEEE, 42(12), 142-150.

7. Razavi, B. (1998). RF microelectronics. Prentice-Hall.

8. Proakis, J. G., \& Salehi, M. (2002). Communication systems engineering (2nd ed.). Prentice Hall.

9. Karlsson, G., Lindfors, S., Skoglund, M., \& Oien, G. Cross-layer optimization in short-range wireless sensor networks (CROPS) a Nordic Research Project Proposal.

10. Shih, E., Cho, S. H., Ickes, N., Min. R., Sinha, A., Wang, A., \& Chandrakasan, A. (2001). Physical layer driven protocol and algorithm design for energy-efficient wireless sensor networks. In MOBICOM'01, July 2001, pp. 272-287.

11. Asada, G. et al. (1998). Wireless integrated network sensors: Low power systems on a chip. In Proc. ESSCIRC'98, September 1998, pp. 9-16.

12. Rabaey, J. et al. (2000). PicoRadio supports ad hoc-ultra-low power wireless networking. Computer, 33(7), 42-48.

13. ADS Ptolemy—eesof.tm.agilent.com/docs/adsdoc2004A/pdf/ptol emy.pdf.

14. Chithrupa, R., Rusu, A., Ismail, M., \& Skoglund, M. (2007) TrACS: Transceiver architecture and wireless channel simulator. In SBCCI 2007, pp. 128-132.

15. Meyr, H., Moeneclaey, M., \& Fechtel, S. A. (1998). Digital communication receivers: Synchronization, channel estimation and signal processing. Wiley Interscience.

16. Rhodes, S. A. (1974). Effect of noisy phase reference on coherent detection of offset-QPSK signals. IEEE Transactions on Communications, COM-22(8), 1046-1055.

17. IEEE Standards 802 Part 15.4 (2003). Wireless medium access control (MAC) and physical layer (PHY) specifications for lowrate wireless personal area networks (LR-WPANs). IEEE Computer Society.

18. Fan, F., \& Li, L.M. (1990). Effect of noisy phase reference on coherent detection of band-limited offset-QPSK signals. IEEE Transactions on Communications, COM-38(2), 156-159.

19. Moeneclaey, M. (1984). The influence of four types of symbol synchronizers on the error probability of a PAM receiver. IEEE Transactions on Communications, COM-32(11), 1186-1190.

20. Prabhu, V. K. (1978). Imperfect carrier recovery effect on filtered PSK signals. IEEE Transactions on Aerospace and Electronic Systems, AES-14(4), 608-615.

21. Liskov, N., \& Curtis, R. (1987). Performance of coherent phase and amplitude digital modulations with carrier recovery noise. IEEE Transactions on Communications, COM-35(9), 972-976.

22. Park, S. B., \& Ismail, M. (2006). DC Offsets in direct conversion multistandard wireless receivers: Modeling and cancellation. Analog Integrated Circuits and Signal Processing, 49(2), 123-130.

23. Enz, C. C., El-Hoiydi, A., Decotignie, J. D., \& Peiris, V. (2004). WiseNET: An ultralow-power wireless sensor network solution. Computer, 37(8), 62-70.

24. Enz C. C., Scolari, N., \& Yodprasit, U. (2005). Ultra low-power radio design for wireless sensor networks. IEEE International Workshop on Radio-Frequency Integration Technology, 1-17.

25. Shinagawa, M., Akazawa, Y., \& Wakimoto, T. (1990). Jitter analysis of high-speed sampling systems. IEEE Journal of SolidState Circuits, 25(1), 224-226.

26. Sun, Y. R., \& Signell, S. (2004). Effects of noise and jitter on algorithms for bandpass sampling in radio receivers. Proceedings of the International Symposium on Circuits and Systems, 1, 208211.

27. Pekau, H., \& Haslett, J. W. (2005). A comparison of analog front end architectures for digital receivers. In IEEE CCECE/CCGEI, Saskatoon, pp. 1073-1077. 


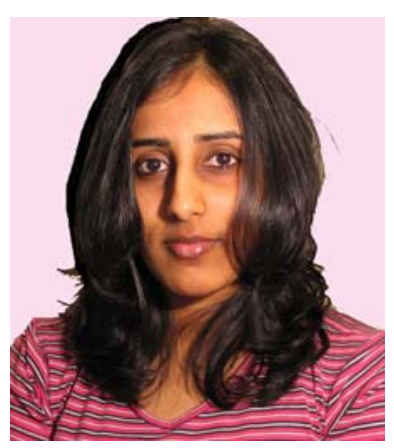

Chithrupa Ramesh received the B.E degree in Telecommunication Engineering from Visvesvarayah Technological University, India in 2004. She is currently working towards the M.S. degree in Electrical Engineering, with a specialization in Wireless Systems at The Royal Institute of Technology (KTH), Stockholm. Her research interests include communication theory and signal processing.

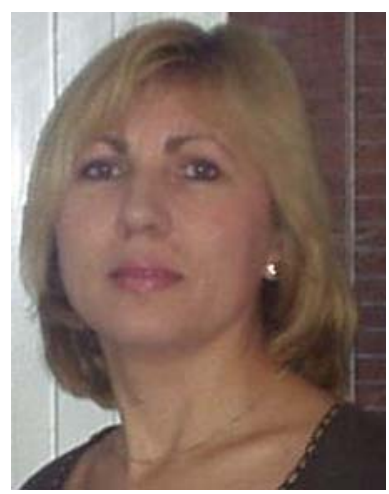

Ana Rusu received degrees of Diploma Engineer (M. S. degree) in Electronics and Telecommunications Engineering from Technical University of Iasi, Romania, in 1983, Ph.D. in Electronics Engineering from Technical University of ClujNapoca, Romania, in 1998 and Docent in Circuit Theory from Royal Institute of Technology Stockholm, Sweden in 2006. Dr. Ana Rusu has over 20 years experience in the field of analog and mixed-signal circuits and systems and she has held several positions in both industry and academia. Since September 2001, she has been with the Royal Institute of Technology (KTH), Stockholm, Sweden, where she is a senior researcher in radio and mixed-signal systems group. Her research interests include data conversion techniques; CAD tools for wireless communications; digitally-enhanced analog/RF front-ends; low-voltage low-power RF, analog and mixed-signal design; Software Defined Radio technology; wireless sensor networks. She has participated in several national and international research projects and has authored or coauthored more than 80 international scientific publications in journals, books, book chapters and conference proceedings.

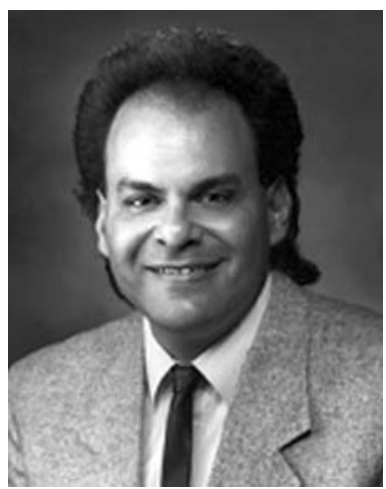

Mohammed Ismail received the B.S. and M.S. degrees in Electronics and Telecommunications Engineering from Cairo University, Egypt, in 1974 and 1978 and the Ph.D. in Electrical Engineering from the University of Manitoba, Canada, in 1983. He is a Professor with the Department of Electrical Engineering, The Ohio State University, Columbus. Since April 2003, he is also a Professor with the Department of Microelectronics and Information Technology, Royal Institute of Technology (KTH) Stockholm, Sweden. He has over 20 years experience of R\&D in the fields of analog, RF and mixed signal integrated circuits. He has held several positions in both industry and academia and has served as a corporate consultant to nearly 30 companies in the US, Europe and the Far East. His current interest lies in research involving digitally programmable/configurable fully integrated radios with focus on low voltage/low power first-pass solutions for $3 \mathrm{G}$ and $4 \mathrm{G}$ wireless handhelds. $\mathrm{He}$ publishes intensively in this area and has been awarded 11 patents. $\mathrm{He}$ has co edited and coauthored several books. He co-founded ANACADEgypt (now part of Mentor Graphics, Inc.) and Spirea AB, Stockholm (now Firstpass Semiconductors AB), a developer of CMOS radio and mixed signal IPs for handheld wireless applications. Dr. Ismail has been the recipient of several awards including the US National Science Foundation Presidential Young Investigator Award, the US Semiconductor Research Corp Inventor Recognition Awards in 1992 and 1993, and a Fulbright/Nokia fellowship Award in 1995. He is the founder of the International Journal of Analog Integrated Circuits and Signal Processing, Springer and serves as the Journal's Editor-In-Chief. He has served as Associate Editor for many IEEE Transactions, was on the Board of Governors of the IEEE Circuits and Systems Society and is the Founding Editor of "The Chip" a Column in The IEEE Circuits and Devices Magazine. He is a Fellow of IEEE.

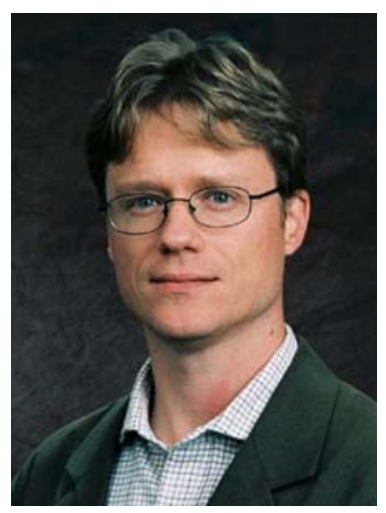

Mikael Skoglund received the $\mathrm{Ph} . \mathrm{D}$. degree in 1997 from Chalmers University of Technology, Göteborg, Sweden. In 1997, he joined the Royal Institute of Technology, Stockholm, Sweden. Here he held various positions until he was appointed Professor of Communication Theory in October 2003. Dr. Skoglund's research interests are in information theory, communications, and detection and estimation. He has worked on problems in source-channel coding, coding and transmission for wireless communications, Shannon theory and statistical signal processing. He has authored more than 110 scientific papers, including papers that have received awards, invited conference presentations, and papers ranking as highly cited according to the ISI Essential Science Indicators. He has also consulted for industry, and he holds six patents. Dr. Skoglund is an Associate Editor with the IEEE Transactions of Communications. 